\title{
Cauer Ladder Network With Multiple Expansion Points for Efficient Model Order Reduction of Eddy-Current Field
}

\section{$\operatorname{AUTHOR}(\mathrm{S})$ :}

Kuriyama, Kenta; Kameari, Akihisa; Ebrahimi, Hassan; Fujiwara, Takayuki; Sugahara, Kengo; Shindo, Yuji; Matsuo, Tetsuji

\section{CITATION:}

Kuriyama, Kenta ...[et al]. Cauer Ladder Network With Multiple Expansion Points for Efficient Model Order Reduction of Eddy-Current Field. IEEE Transactions on Magnetics 2019, 55(6): 7203404.

\section{ISSUE DATE:}

2019-06

URL:

http://hdl.handle.net/2433/244835

\section{RIGHT:}

C 2019 IEEE. Personal use of this material is permitted. Permission from IEEE must be obtained for all other uses, in any current or future media, including reprinting/republishing this material for advertising or promotional purposes, creating new collective works, for resale or redistribution to servers or lists, or reuse of any copyrighted component of this work in other works.; この論文は出版社版でありません。引用の際には出版社版をご確認ご利用ください。; This is not the published version. Please cite only the published version. 


\title{
Cauer Ladder Network with Multiple Expansion Points for Efficient Model Order Reduction of Eddy-Current Field
}

\author{
Kenta Kuriyama ${ }^{1}$, Akihisa Kameari ${ }^{2}$, Hassan Ebrahimi ${ }^{2}$, Takayuki Fujiwara ${ }^{1}$, \\ Kengo Sugahara $^{3}$, Yuji Shindo ${ }^{4}$ and Tetsuji Matsuo ${ }^{1}$ \\ ${ }^{1}$ Graduate School of Engineering, Kyoto University, Kyoto 615-8510, Japan \\ ${ }^{2}$ Science Solutions International Laboratory, Inc., Tokyo 153-0065, Japan \\ ${ }^{3}$ Faculty of Science and Engineering, Kindai University, Higashi-Osaka 577-8502, Japan \\ ${ }^{4}$ Kawasaki Heavy Industries, Ltd., Akashi 673-8666, Japan
}

\begin{abstract}
The Cauer ladder network (CLN) method provides an efficient representation of eddy-current fields. This paper proposes a reformulation of the CLN method that considers expansion points using the magnetic/current vector potential. To cover a wide range of frequencies, the expansion point may be changed at a later stage of the network. Only a few stages of the ladder network are required for the eddy-current field to be reconstructed accurately around the target frequency using the expansion points.
\end{abstract}

Index Terms-Cauer ladder network, model order reduction, multiple expansion points, magnetic/current vector potential.

\section{INTRODUCTION}

$\mathbf{T}$ HE control of power by high-frequency switching is widely used these days but requires an efficient analytical method to calculate the electromagnetic fields present when handling small skin depths and small time steps. To avoid direct large-scale computations, model order reduction (MOR) methods [1]-[4] have been intensively studied recently in an effort to approximate accurately large-scale systems with corresponding reduced-scale systems.

The Cauer ladder network (CLN) [5][6] is an efficient MOR method that replaces eddy-current fields by an equivalent electric circuit/network based on an orthogonal expansion of the electric and magnetic fields. Adopting the finite-element method (FEM), a matrix formulation [7] is derived to clarify the theoretical aspects of the CLN method. The CLN method was originally developed with an expansion point at zero frequency, implying that the number of network stages becomes large when the target frequency is high.

This paper formulates a CLN method that considers nonzero expansion points as exploited in other MOR methods [1][2][3]. First, the CLN method with a single expansion point is developed based on the FEM using the magnetic vector potential. This CLN method is extended to multiple expansion points to cover the range of target frequencies with a minimum number of network elements; here, the expansion point is changed at a later stage in the network. This paper also presents another CLN formulation using the current vector potential that yields a different type of network.

\section{FORMULATION OF CLN WITH EXPANSION POINTS}

\section{A. Magnetic Vector Potential Formulation}

In finite-element space, the magnetic vector potential $\boldsymbol{A}$ and the electric field $\boldsymbol{E}$ are discretized as prescribed by

$$
\boldsymbol{A}=\sum_{i} a_{i} \boldsymbol{w}_{i}^{1}, \quad \boldsymbol{E}=\sum_{i} e_{i} \boldsymbol{w}_{i}^{1}
$$

where $\boldsymbol{w}_{i}^{1}$ is an edge element [8]. From the coefficients of (1), coordinate vectors $\boldsymbol{a}$ and $\boldsymbol{e}$ are defined,

$$
\boldsymbol{a}=\left[a_{1}, a_{2}, \ldots\right]^{\mathrm{T}}, \boldsymbol{e}=\left[e_{1}, e_{2}, \ldots\right]^{\mathrm{T}} .
$$

The reluctivity and conductivity matrices are defined in the domain $\Omega$ as follows:

$$
\begin{array}{ll}
\boldsymbol{\nu}=\left\{\nu_{i j}\right\}, & \nu_{i j}=\int_{\Omega} \frac{1}{\mu} \boldsymbol{w}_{i}^{2} \cdot \boldsymbol{w}_{j}^{2} d \Omega, \\
\boldsymbol{\sigma}=\left\{\sigma_{i j}\right\}, & \sigma_{i j}=\int_{\Omega} \sigma \boldsymbol{w}_{i}^{1} \cdot \boldsymbol{w}_{j}^{1} d \Omega
\end{array}
$$

where $\mu, \sigma$, and $\boldsymbol{w}_{i}^{2}$ denote the permeability, conductivity, and the $i$-th face element [8].

The governing equation of the eddy-current field using the A-formulation is represented in the form

$$
\boldsymbol{C}^{\mathrm{T}} \boldsymbol{\nu} \boldsymbol{C a}=-j \omega \boldsymbol{\sigma a}+\boldsymbol{j}_{0},
$$

where $\boldsymbol{C}$ represents an edge-face incident matrix [8], and $\boldsymbol{j}_{0}$ the discretized source current density. The boundary condition depends on the power source.

For the CLN method with expansion points, and using the A-formulation, the orthogonal basis vectors $\left\{\boldsymbol{e}_{2 n}\right\}$ and $\left\{\boldsymbol{a}_{2 n+1}\right\}$ are generated by solving recursive equations.

Consider expansion point $s_{i}=2 \pi f_{i}$; its corresponding coefficient matrix $\boldsymbol{K}_{i}$ is defined as

$$
\boldsymbol{K}_{i}=\boldsymbol{C}^{\mathrm{T}} \boldsymbol{\nu} \boldsymbol{C}+s_{i} \boldsymbol{\sigma} .
$$

Using $\boldsymbol{K}_{\mathbf{0}}$, the CLN procedure for a single expansion point $s_{0} \neq 0$ is [see Fig. 1] similar to the original CLN with $s_{0}=0$.

A unit voltage/current source is given to start the CLN procedure. Suppose the electric field $-\operatorname{grad} \varphi$ due to unit voltage is a source term in a domain of a conductor; the equation

$$
\boldsymbol{K}_{0} \boldsymbol{a}^{\prime}=-\boldsymbol{\sigma} \boldsymbol{G} \boldsymbol{\varphi}
$$

is first solved to determine the initial electric field (8),

$$
\boldsymbol{e}_{0}=-\boldsymbol{G} \boldsymbol{\varphi}-s_{0} \boldsymbol{a}^{\prime}
$$

where $\varphi$ denotes a vector representing the scalar potential, and $\boldsymbol{G}$ is the node-edge incidence matrix, which satisfies $\boldsymbol{C} \boldsymbol{G}=0$. When a Dirichlet boundary condition on $\boldsymbol{E}$ or $\boldsymbol{A}$ is given, the equation for $\boldsymbol{a}_{2 n+1}$ is solved subject to the constraint that the corresponding boundary components of $\boldsymbol{a}_{2 n+1}$ vanish. When the current density $\boldsymbol{j}_{0}$ due to unit current is imposed, the initial condition is given as $\boldsymbol{e}_{0}=0$ and $\boldsymbol{a}_{1}=\boldsymbol{K}_{0}^{-1} \boldsymbol{j}_{0}$.

Setting $R_{2 n}=1 / \lambda_{2 n}$ and $L_{2 n+1}=\lambda_{2 n+1}$, the Cauer 


$$
\begin{aligned}
& \text { solve }: K_{0} a^{\prime}=-\sigma G \varphi \\
& \boldsymbol{e}_{0}=-\boldsymbol{G} \boldsymbol{\varphi}-s_{0} \boldsymbol{a}^{\prime} \\
& \lambda_{-1}=\frac{1}{\boldsymbol{e}_{0}^{T} \boldsymbol{K}_{0} \boldsymbol{e}_{0}}, \boldsymbol{a}_{-1}^{*}=-\lambda_{-1} \boldsymbol{e}_{0}, \boldsymbol{a}_{-1}=0, n=0 \\
& \text { *Loop } \quad \lambda_{2 n}=\boldsymbol{e}_{2 n}^{T} \boldsymbol{\sigma} \boldsymbol{e}_{2 n} \leftarrow n \leftarrow n+1 \\
& \text { solve }: \boldsymbol{K}_{0}\left(\boldsymbol{a}_{2 n+1}-\boldsymbol{a}_{2 n-1}\right)=\frac{1}{\lambda_{2 n}} \boldsymbol{\sigma} \boldsymbol{e}_{2 n} \\
& \lambda_{2 n+1}=\boldsymbol{a}_{2 n+1}^{T} \boldsymbol{K}_{0} \boldsymbol{a}_{2 n+1} \\
& \boldsymbol{e}_{2 n+2}-\boldsymbol{e}_{2 n}=-\frac{1}{\lambda_{2 n+1}} \boldsymbol{a}_{2 n+1}
\end{aligned}
$$

Fig. 1. Flowchart for the A-formulation.

equivalent circuit for the single expansion point $s_{0}$ is constructed [Fig. 2] in a similar way to the original CLN [7].

The expansion point may be changed at a later stage of the circuit. Consider now two expansion points $s_{0}$ and $s_{1}$; then $\left\{\boldsymbol{e}_{2 n}\right\}$ and $\left\{\boldsymbol{a}_{2 n+1}\right\}$, which were changed at the $M+1$-th stage, are generated in the following procedure.

\section{Step 1:}

Using the procedure in Fig. 1, solve equations with $s_{0}$ for $0 \leq n \leq M-2$ and generate $\boldsymbol{e}_{0}, \boldsymbol{e}_{2}, \ldots, \boldsymbol{e}_{2 M-2}$, and $\boldsymbol{a}_{-1}^{*}, \boldsymbol{a}_{1}, \ldots, \boldsymbol{a}_{2 M-3}$.

\section{Step 2:}

$$
\begin{aligned}
& \text { For } n=M-1, \text { solve } \\
& \qquad \begin{array}{l}
\boldsymbol{K}_{1} \boldsymbol{a}_{2 M-1}-\boldsymbol{K}_{0} \boldsymbol{a}_{2 M-3}=\frac{1}{\lambda_{2 M-2}} \boldsymbol{\sigma} \boldsymbol{e}_{2 M-2}, \\
\boldsymbol{e}_{2 M}-\boldsymbol{e}_{2 M-2}=-\frac{1}{\lambda_{2 M-1}}\left(\boldsymbol{a}_{2 M-1}+\sum_{i=1}^{M-1} p_{2 i-1} \boldsymbol{a}_{2 i-1}\right)
\end{array}
\end{aligned}
$$

and generate $\boldsymbol{a}_{2 M-1}$ and $\boldsymbol{e}_{2 M} \cdot \lambda_{2 M-1}$ and $\lambda_{2 M}$ are calculated by

$$
\begin{aligned}
& \lambda_{2 M-1}=\boldsymbol{a}_{2 M-1}^{\mathrm{T}} \boldsymbol{K}_{1} \boldsymbol{a}_{2 M-1}, \\
& \lambda_{2 M}=\boldsymbol{e}_{2 M}^{\mathrm{T}} \boldsymbol{\sigma} \boldsymbol{e}_{2 M} .
\end{aligned}
$$

To satisfy the orthogonality between $\left\{\boldsymbol{e}_{2 M}\right\}$ and $\left\{\boldsymbol{e}_{2 n}\right\}$ $(0 \leq n \leq M-1), p_{2 i-1}(1 \leq i \leq M-1)$ is determined as follows:

$$
p_{2 i-1}=-\frac{1}{\lambda_{2 i-1}} \boldsymbol{a}_{2 M-1}^{\mathrm{T}} \boldsymbol{K}_{0} \boldsymbol{a}_{2 i-1} \text {. }
$$

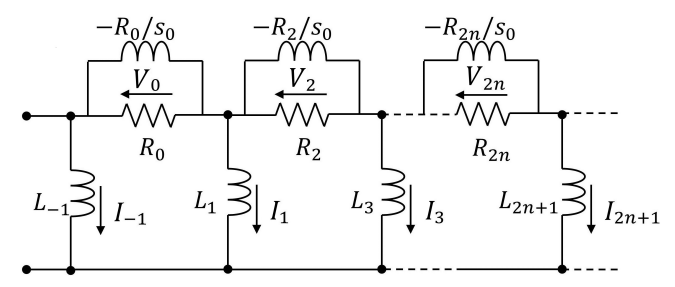

Fig. 2. Circuit with single expansion point $s_{0}$ (A-fomulation).

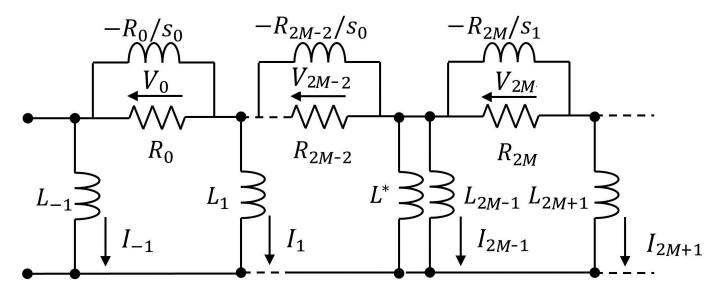

Fig. 3. Circuit with two expansion points $s_{0}$ and $s_{1}$ (A-fomulation).

\section{Step 3:}

Replacing $\boldsymbol{K}_{0}$ by $\boldsymbol{K}_{1}$ in Fig. 1, solve equations with $s_{1}$ for $n \geq M$ in the loop and generate $\boldsymbol{a}_{2 M+1}, \ldots$ and $\boldsymbol{e}_{2 M+2}, \ldots$

Using $\boldsymbol{a}_{2 M-1}^{\prime}$ defined as

$$
\boldsymbol{a}_{2 M-1}^{\prime}=\boldsymbol{a}_{2 M-1}+\sum_{i=1}^{M-1} p_{2 i-1} \boldsymbol{a}_{2 i-1}
$$

the electromagnetic fields $\boldsymbol{e}$ and $\boldsymbol{a}$ are expanded in the form

$$
\begin{aligned}
& \boldsymbol{e}=\sum_{n=0} V_{2 n} \boldsymbol{e}_{2 n}, \\
& \boldsymbol{a}=\sum_{\substack{n=0 \\
n \neq M-1}} I_{2 n+1} \boldsymbol{a}_{2 n+1}+I_{2 M-1} \boldsymbol{a}_{2 M-1}^{\prime}+I_{-1} \boldsymbol{a}_{-1}^{*} .
\end{aligned}
$$

Given the expansions (15) and (16), a network expression [Fig. 3] is derived in a similar manner as for the original CLN. Given that $\boldsymbol{a}_{2 M-3}^{T} \boldsymbol{K}_{1} \boldsymbol{a}_{2 M-1}^{\prime} \neq 0$, an additional inductor is required, the inductance $L^{*}$ of which is evaluated using

$$
L^{*}=-\frac{\lambda_{2 M-1}}{\left(s_{1}-s_{0}\right) \lambda_{2 M-2}\left(\lambda_{2 M-1}+p_{2 M-3} \lambda_{2 M-3}\right)} .
$$

A further change of the expansion point is possible as performed similarly in the above.

\section{B. Current Vector Potential Formulation}

Next a CLN method is described which uses the current vector potential to handle only conductive materials for simplicity. The current vector potential $\boldsymbol{T}$ and magnetic field $\boldsymbol{H}$ are first discretized,

$$
\boldsymbol{T}=\sum_{i} t_{i} \boldsymbol{w}_{i}^{1}, \quad \boldsymbol{H}=\sum_{i} h_{i} \boldsymbol{w}_{i}^{1}
$$

From the coefficients, coordinate vectors $\boldsymbol{t}$ and $\boldsymbol{h}$ are defined,

$$
\boldsymbol{t}=\left[t_{1}, t_{2}, \ldots\right]^{\mathrm{T}}, \boldsymbol{h}=\left[h_{1}, h_{2}, \ldots\right]^{\mathrm{T}} .
$$

The resistivity and permeability matrices are defined for the domain $\Omega$,

$$
\begin{array}{ll}
\boldsymbol{\rho}=\left\{\rho_{i j}\right\}, \quad \rho_{i j}=\int_{\Omega} \frac{1}{\sigma} \boldsymbol{w}_{i}^{2} \cdot \boldsymbol{w}_{j}^{2} d \Omega, \\
\boldsymbol{\mu}=\left\{\mu_{i j}\right\}, \quad \mu_{i j}=\int_{\Omega} \mu \boldsymbol{w}_{i}^{1} \cdot \boldsymbol{w}_{j}^{1} d \Omega .
\end{array}
$$

The governing equation of the eddy-current field in the T-formulation has the form

$$
\boldsymbol{C}^{\mathrm{T}} \boldsymbol{\rho} \boldsymbol{C t}=-j \omega \boldsymbol{\mu t} .
$$

The orthogonal basis vectors $\left\{\boldsymbol{t}_{2 n}\right\}$ and $\left\{\boldsymbol{h}_{2 n+1}\right\}$ are generated as follows.

Considering expansion point $s_{i}=2 \pi f_{i}$, the associated coefficient matrix $\boldsymbol{K}_{i}$ is defined as

$$
\boldsymbol{K}_{i}=\boldsymbol{C}^{\mathrm{T}} \boldsymbol{\rho} \boldsymbol{C}+s_{i} \boldsymbol{\mu} .
$$

Using $\boldsymbol{K}_{\mathbf{0}}$, the CLN procedure for a single expansion point $s_{0} \neq 0$ is derived in a similar manner as for the original CLN [see Fig. 4].

With a Neumann boundary condition on $\boldsymbol{T}$ imposed at the corresponding boundary $\partial \Omega$ by the power source, the equation for $t_{2 n}$ is solved subject to the condition

$$
\boldsymbol{\rho} \boldsymbol{C}\left(\boldsymbol{t}_{2 n+2}-\boldsymbol{t}_{2 n}\right)=0 \text { at } \partial \Omega .
$$




$$
\begin{gathered}
\text { solve }: \boldsymbol{K}_{0} \boldsymbol{t}_{0}=0 \\
\lambda_{0}=\boldsymbol{t}_{0}^{T} \boldsymbol{K}_{0} \boldsymbol{t}_{0}, \boldsymbol{h}_{1}=\frac{1}{\lambda_{0}} \boldsymbol{t}_{0}, n=0 \\
\text { *Loop } \lambda_{2 n+1}=\boldsymbol{h}_{2 n+1}^{T} \boldsymbol{\mu} \boldsymbol{h}_{2 n+1} \\
\hline \text { solve : } \boldsymbol{K}_{0}\left(\boldsymbol{t}_{2 n+2}-\boldsymbol{t}_{2 n}\right)=-\frac{1}{\lambda_{2 n+1}} \boldsymbol{\mu} \boldsymbol{h}_{2 n+1} \\
\lambda_{2 n+2}=\boldsymbol{t}_{2 n+2}^{T} \boldsymbol{K}_{0} \boldsymbol{t}_{2 n+2} \\
\boldsymbol{h}_{2 n+3}-\boldsymbol{h}_{2 n+1}=\frac{1}{\lambda_{2 n+2}} \boldsymbol{t}_{2 n+2}
\end{gathered}
$$

Fig. 4. Flowchart for the T-formulation.

A CLN with a single expansion point $s_{0}$ is constructed [Fig. 5].

Consider two expansion points $s_{0}$ and $s_{1}$ changed at the $M+1$-th stage; then $\left\{\boldsymbol{t}_{2 n}\right\}$ and $\left\{\boldsymbol{h}_{2 n+1}\right\}$ are generated in the following procedure.

\section{Step 1:}

Using the procedure given in Fig. 4, solve equations with $s_{0}$ for $0 \leq n \leq M-2$ and generate $\boldsymbol{t}_{0}, \boldsymbol{t}_{2}, \ldots, \boldsymbol{t}_{2 M-2}$ and $\boldsymbol{h}_{1}, \boldsymbol{h}_{3}, \ldots, \boldsymbol{h}_{2 M-1}$.

\section{Step 2:}

For $n=M-1$, solve

$$
\begin{aligned}
& \boldsymbol{K}_{1} \boldsymbol{t}_{2 M}-\boldsymbol{K}_{0} \boldsymbol{t}_{2 M-2}=-\frac{1}{\lambda_{2 M-1}} \boldsymbol{\mu} \boldsymbol{h}_{2 M-1}, \\
& \boldsymbol{h}_{2 M+1}-\boldsymbol{h}_{2 M-1}=\frac{1}{\lambda_{2 M}}\left(\boldsymbol{t}_{2 M}+\sum_{i=0}^{M-1} p_{2 i} \boldsymbol{t}_{2 i}\right)
\end{aligned}
$$

and generate $\boldsymbol{t}_{2 M}$ and $\boldsymbol{h}_{2 M+1} \cdot \lambda_{2 M}$ and $\lambda_{2 M+1}$ are calculated from

$$
\begin{aligned}
& \lambda_{2 M}=\boldsymbol{t}_{2 M}^{\mathrm{T}} \boldsymbol{K}_{1} \boldsymbol{t}_{2 M}, \\
& \lambda_{2 M+1}=\boldsymbol{h}_{2 M+1}^{\mathrm{T}} \boldsymbol{\mu} \boldsymbol{h}_{2 M+1} .
\end{aligned}
$$

For orthogonality to hold between $\left\{\boldsymbol{h}_{2 M+1}\right\}$ and $\left\{\boldsymbol{h}_{2 n+1}\right\}$ $(0 \leq n \leq M-1), p_{2 i}(0 \leq i \leq M-1)$ must be set to

$$
p_{2 i}=-\frac{1}{\lambda_{2 i}} \boldsymbol{t}_{2 M}^{\mathrm{T}} \boldsymbol{K}_{0} \boldsymbol{t}_{2 i} \text {. }
$$

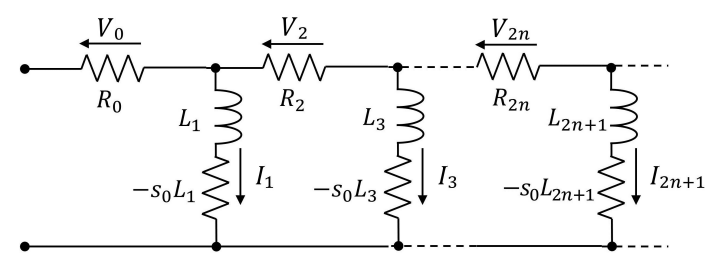

Fig. 5. Circuit with a single expansion point $s_{0}$ (T-formulation).

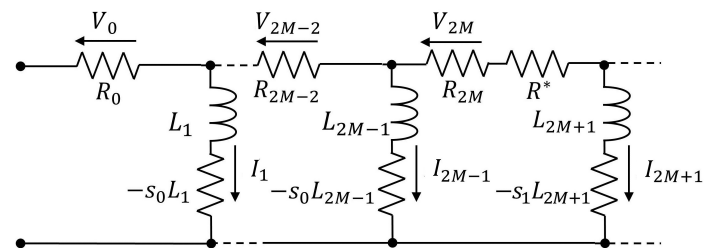

Fig. 6. Circuit with two expansion points $s_{0}$ and $s_{1}$ (T-formulation).
Step 3:

Replacing $\boldsymbol{K}_{0}$ by $\boldsymbol{K}_{1}$ in Fig. 4, solve equations with $s_{1}$ for $n \geq M$ in the loop and generate $\boldsymbol{t}_{2 M+2}, \ldots$ and $\boldsymbol{h}_{2 M+3}, \ldots$.

Using $\boldsymbol{t}_{2 M}^{\prime}$ defined as

$$
\boldsymbol{t}_{2 M}^{\prime}=\boldsymbol{t}_{2 M}+\sum_{i=0}^{M-1} p_{2 i} \boldsymbol{t}_{2 i}
$$

the electromagnetic fields $t$ and $\boldsymbol{h}$ are expanded as

$$
\begin{aligned}
\boldsymbol{t} & =\sum_{\substack{n=0 \\
n \neq M}} V_{2 n} \boldsymbol{t}_{2 n}+V_{2 M} \boldsymbol{t}_{2 M}^{\prime}, \\
\boldsymbol{h} & =\sum_{n=0} I_{2 n+1} \boldsymbol{h}_{2 n+1} .
\end{aligned}
$$

With these expansions (31) and (32), a network expression [Fig. 6] is derived in similar manner to the original CLN. Because $\boldsymbol{t}_{2 M-2}^{T} \boldsymbol{K}_{1} \boldsymbol{t}_{2 M}^{\prime} \neq 0$, an additional resistor is required having resistance $R^{*}$

$$
R^{*}=-\frac{\left(s_{1}-s_{0}\right) \lambda_{2 M-1}\left(\lambda_{2 M}+p_{2 M-2} \lambda_{2 M-2}\right)}{\lambda_{2 M}} .
$$

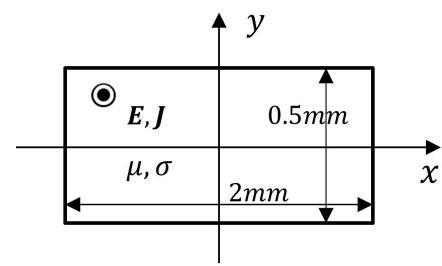

Fig. 7. Iron bar with a rectangular cross-section.
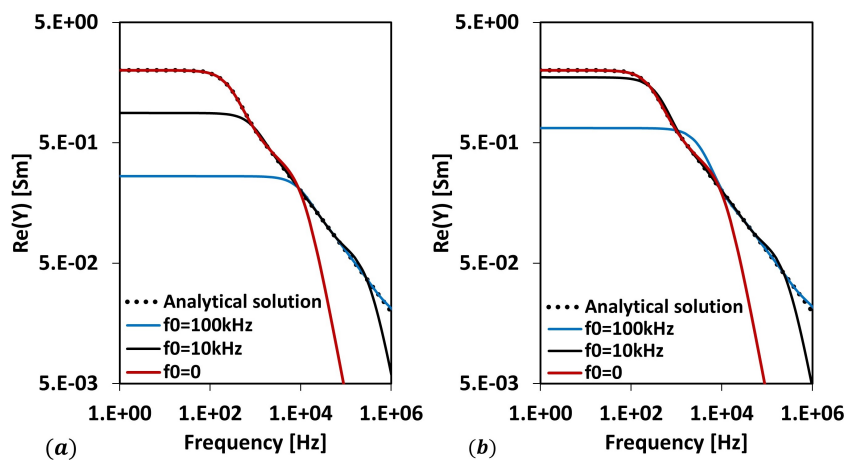

Fig. 8. Real part of the admittance per unit length represented at the 4-th stage with a single expansion point $s_{0}=2 \pi f_{0}$ : (a) A-formulation, and (b) T-formulation.
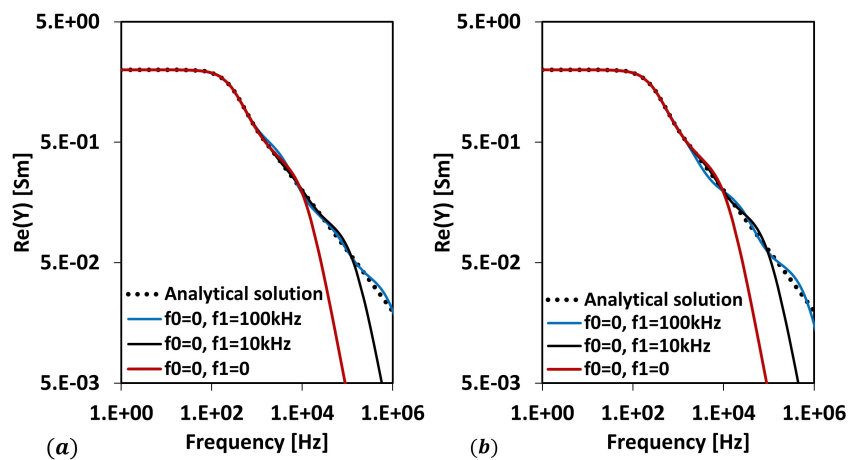

Fig. 9. Real part of the admittance per unit length represented by 4-stage with two expansion points $s_{0}=0$ and $s_{1}=2 \pi f_{1}$ : (a) A-formulation, and (b) T-formulation. 


\section{NUMERICAL CALCULATION}

\section{A. Iron bar with rectangular cross-section}

The CLN methods with both the A- and T-formulations are applied to an iron bar with rectangular cross-section [Fig. 7], the conductivity of the bar being $2 \times 10^{6} \mathrm{~S} / \mathrm{m}$ and the permeability is $0.01 \mathrm{H} / \mathrm{m}$. A unit electric field is given at the surface of the iron bar. The analytical solution is obtained by applying a Fourier expansion. The real part of the admittance per unit length [Fig. 8] is represented by the 4-stage CLN obtained with a single expansion point $s_{0}=2 \pi f_{0}$, with $f_{0}=0,10 \mathrm{kHz}, 100 \mathrm{kHz}$. The frequency dependence is reproduced accurately around the expansion points. The frequency dependence obtained with two expansion points $s_{0}=0$ and $s_{1}=2 \pi f_{1}$ [Fig. 9] shows the effect of one expansion point being changed at the third stage to $f_{1}=10 \mathrm{kHz}$ and $100 \mathrm{kHz}$. A wide frequency range is covered by the two expansion points.

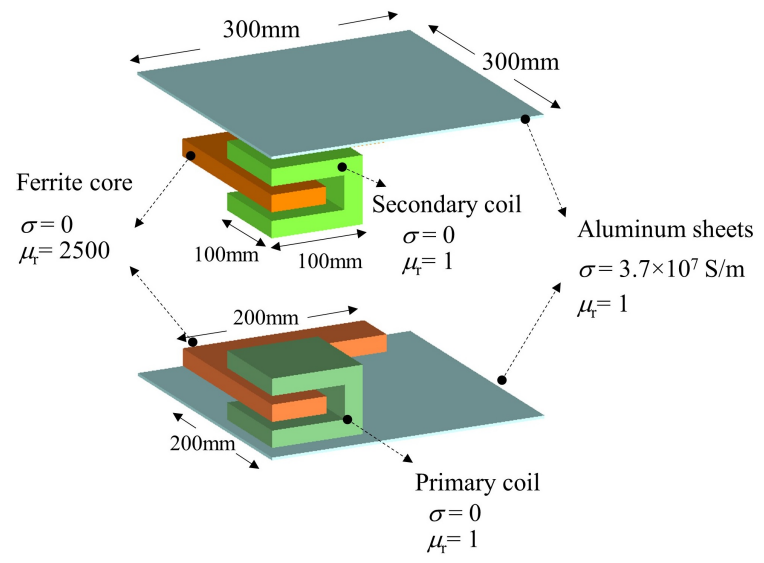

Fig. 10. Wireless power transfer system.

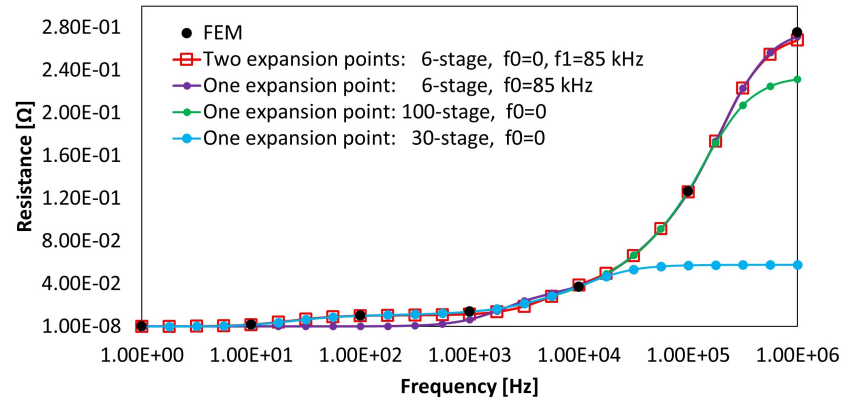

Fig. 11. Frequency dependence of resistance $\operatorname{Re}(Z)$.

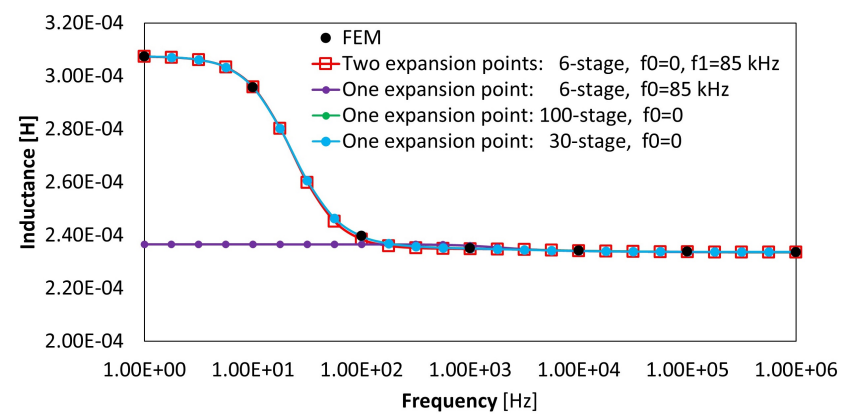

Fig. 12. Frequency dependence of inductance $\operatorname{Im}(Z) / \omega$.

\section{B. Wireless power transfer system}

A wireless power transfer system [Fig. 10] was analyzed. The primary coil has ten turns and the secondary coil is open. Its working frequency is $85 \mathrm{kHz}$. The frequency dependence of its impedance $Z$ seen from the primary coil was plotted [Figs. 11 and 12]. When the single expansion point $s_{0}$ is zero, the impedance is not accurate even after 100 stages of the CLN. The single expansion point of $85 \mathrm{kHz}$ achieves an accurate impedance around the expansion point with a small number of stages. Using two expansion points of $\left(s_{0}, s_{1}\right)=2 \pi \times(0,85) \mathrm{kHz}$, an accurate impedance was obtained from 0 to $1 \mathrm{MHz}$ only with six stages of the CLN, where the expansion point is changed at the second stage.

\section{CONCLUSION}

A CLN method with expansion points was developed using the magnetic/current vector potential. Multiple expansion points were introduced by changing them at a later stage of the circuit while retaining field orthogonality. The proposed methods accurately replace the eddy-current fields by small scale CLNs around the expansion points. Because the area of application of the T-formulation is limited, a $\mathrm{T}-\Omega$ version of the CLN method should be developed as well as the A- $\phi$ version.

\section{ACKNOWLEDGMENT}

We thank Richard Haase, Ph.D, from Edanz Group (www.edanzediting.com/ac) for editing a draft of this manuscript.

\section{REFERENCES}

[1] P. Feldmann and R. W. Freund : "Efficient Linear Circuit Analysis by Padé Approximation via the Lanczos Process”, IEEE Trans. Comput-Aided Design Integr Circuits Syst., Vol. 14, No. 5, pp.639-649, May. 1995.

[2] A. Pierquin, T. Henneron, S. Clénet and S. Brisset : “ Model-Order Reduction of Magnetoquasi-Static Problems Based on POD and Arnoldi-Based Krylov Methods”, IEEE Trans. Magn., Vol.51, No.3, Mar. 2015.

[3] Y. Sato and H. Igarashi : "Generation of Equivalent Circuit From Finite-Element Model Using Model Order Reduction”, IEEE Trans. Magn., Vol.52, No.3, Mar. 2016.

[4] Y. Sato, M. Clemens and H. Igarashi : “Adaptive Subdomain Model Order Reduction With Discrete Empirical Interpolation Method for Nonlinear Magneto-Quasi-Static Problems", IEEE Trans. Magn., Vol.52, No.3, Mar. 2016.

[5] A. Kameari, H. Ebrahimi, K. Sugahara, Y. Shindo and T. Matsuo : "Cauer Ladder Network Representation of Eddy-Current Fields for Model Order Reduction Using Finite Element Method”, IEEE Trans. Magn., Vol.54, No.3, Mar. 2018.

[6] K. Sugahara, A. Kameari, H. Ebrahimi, Y. Shindo and T. Matsuo : "Finite Element Analysis of Unbounded Eddy-Current Problems Using Cauer Ladder Network method”, IEEE Trans. Magn., Vol.54, No.3, Mar. 2018.

[7] T. Matsuo, A. Kameari, K. Sugahara and Y. Shindo: “Matrix Formulation of the Cauer Ladder Network Method for Efficient Eddy-Current Analysis”, IEEE Trans. Magn., Vol.54, No11, Nov. 2018.

[8] A. Bossavit, Computational Electromagnetism, Academic Press, 1998. 